\title{
Further results involving Marshall-Olkin log-logistic distribution: reliability analysis, estimation of the parameter, and applications
}

\author{
Arwa M. Alshangiti", M. Kayid and B. Alarfaj
}

*Correspondence: arwash@ksu.edu.sa Department of Statistics and Operations Research, College of Science, King Saud University, P.O. Box 2455 Riyadh 11451, Kingdom of Saudi Arabia

\begin{abstract}
The purpose of this paper is to provide further study of the Marshall-Olkin log-logistic model that was first described by Gui (Appl Math Sci 7:3947-3961, 2013). This model is both useful and practical in areas such as reliability and life testing. Some statistical and reliability properties of this model are presented including moments, reversed hazard rate and mean residual life functions, among others. Maximum likelihood estimation of the parameters of the model is discussed. Finally, a real data set is analyzed and it is observed that the presented model provides a better fit than the log-logistic model.
\end{abstract}

Keywords: Marshall-Olkin, Reversed hazard rate function, Mean residual life function, Mean inactivity time function, Maximum likelihood estimates, Applications

\section{Background}

Many researchers strive to introduce new families of distributions or to generalize existing distributions, which can be used to describe the lifetimes of devices or to describe sets of real data. In Marshall and Olkin (1997), Marshall and Olkin (M-O) introduced a new family of distribution in an attempt to add a parameter to a family of distributions. Let $X$ be a random variable with survival function $\bar{F}(x)=1-F(x)$, then

$$
\begin{aligned}
\bar{G}(x ; \alpha) & =\frac{\alpha \bar{F}(x)}{1-\bar{\alpha} \bar{F}(x)} \\
& =\frac{\alpha \bar{F}(x)}{F(x)+\alpha \bar{F}(x)}, \quad-\infty \leq x \leq \infty, \quad \alpha>0,
\end{aligned}
$$

is a proper survival function called $\mathrm{M}-\mathrm{O}$ family of distributions where $\bar{\alpha}=1-\alpha$. Clearly, Eq. (1) provides a tool to obtain a parametric distribution from existing one. The probability density function (pdf) of the general M-O family, say $g(x)$, takes the form

$$
g(x ; \alpha)=\frac{\alpha f(x)}{[1-\bar{\alpha} \bar{F}(x)]^{2}}, \quad-\infty \leq x \leq \infty,
$$

where $f(x)$ is the pdf corresponding to $F(x)$. Applications, properties and applications of M-O extended distributions can be found in Alshangiti et al. $(2014,2016)$, Okasha and

\section{Springer Open}

๑) 2016 Alshangiti et al. This article is distributed under the terms of the Creative Commons Attribution 4.0 International License (http://creativecommons.org/licenses/by/4.0/), which permits unrestricted use, distribution, and reproduction in any medium, provided you give appropriate credit to the original author(s) and the source, provide a link to the Creative Commons license, and indicate if changes were made. 
Kayid (2016), Ghitany et al. (2007), Ristic et al. (2007), El-Bassiouny and Abdo (2009, 2010), Srinivasa et al. (2011), Jose and Krishna (2011), Lin and Li (2012), Cordeiro and Lemonte (2012).

Recently, Gui (2013) introduced and studied the $\mathrm{M}-\mathrm{O} \log$ logistic distribution, denoted by $\mathrm{M}-\mathrm{O}$ log-logistic. The paper's objectives are to investigate some statistical and reliability properties of $\mathrm{M}-\mathrm{O} \log$-logistic distribution and to illustrate its applicability in different areas. The paper is organized into five sections. The density and the moment of the model are given in "Extended log-logistic distribution" section. In that section, we provide some new statistical and reliability functions (reversed hazard rate, mean residual life, mean inactivity time, etc.) and discuss their properties. Furthermore, maximum likelihood estimation problems are considered in "Maximum likelihood estimators" section. To indicate the adequacy of the model, some applications using a numerical example and an example with real data are discussed in "Fitting reliability data" section. Finally, in "Conclusion" section, we provide a brief conclusion and some remarks regarding the current and future research (Additional file 1).

\section{Extended log-logistic distribution}

In probability and statistics, the log-logistic distribution (LLD) (known as the Fisk distribution in economics) is a continuous probability distribution for a non-negative random variable. It is used in survival analysis as a parametric model for events whose rate increases initially and decreases later, for example mortality rate from cancer following diagnosis or treatment. It has also been used in hydrology to model stream flow and precipitation, and in economics as a simple model of the distribution of wealth or income. The LLD is obtained by applying the logarithmic transformation to the logistic distribution in much the same way as the log-normal distribution is obtained from normal distribution or the log-Pearson distribution from the Pearson distribution. The LLD is a special case of Burr's type-XII and also a special case of the Kappa distribution, that have been applied to precipitation (c.f. Burr 1942; Mielke and Johnson 1973). The survival function of the log-logistic distribution $(\beta, \gamma)$ takes the form

$$
\bar{F}(x ; \beta, \gamma)=\frac{1}{1+\left(\frac{x}{\gamma}\right)^{\beta}}, \quad x \geq 0,
$$

where $\gamma>0, \beta>1$. Here $\beta$ is a shape parameter and $\gamma$ is a scale parameter. According to Gui (2013), substituting (3) in (1) we get the $\mathrm{M}-\mathrm{O} \log$ logistic distribution, denoted by $\mathrm{M}-\mathrm{O} \log$-logistic $(\alpha, \beta, \gamma)$ with survival function

$$
\bar{G}(x ; \alpha, \beta, \gamma)=\frac{\alpha}{\alpha+\left(\frac{x}{\gamma}\right)^{\beta}}, \quad 0 \leq x \leq \infty, \quad \alpha, \gamma>0, \quad \beta>1 .
$$

The corresponding CDF and pdf are obtained respectively as

$$
G(x ; \alpha, \beta, \gamma)=\frac{\left(\frac{x}{\gamma}\right)^{\beta}}{\alpha+\left(\frac{x}{\gamma}\right)^{\beta}}, \quad 0 \leq x \leq \infty, \quad \alpha, \gamma>0, \quad \beta>1,
$$


and

$$
g(x ; \alpha, \beta, \gamma)=\frac{\alpha \frac{\beta}{\gamma}\left(\frac{x}{\gamma}\right)^{\beta-1}}{\left[\alpha+\left(\frac{x}{\gamma}\right)^{\beta}\right]^{2}}, \quad 0 \leq x \leq \infty, \quad \alpha, \gamma>0, \beta>1 .
$$

\section{Statistical and reliability properties}

In this subsection, we investigate some statistical and reliability properties of the M-O log-logistic. Let $X \geq 0$ be a random variable representing life with $\mathrm{cdf} G$ and $\mathrm{rf}$ $\bar{G}=1-G$ and assume that $G$ admits the probability density $g$. The reversed hazard rate (RHR) of $X$ is defined by

$$
r_{F}(x)=\frac{g(x)}{G(x)}, \quad x>0 .
$$

The RHR function is well-known and useful tool in reliability theory and in other areas of applied probability and statistics. In addition, the RHR function has been receiving increasing attention in the recent literature of reliability analysis and stochastic modeling. The RHR of a random variable $X$ with $\mathrm{M}-\mathrm{O} \log$-logistic $(\alpha, \beta, \gamma)$ respectively is

$$
r(x ; \alpha, \sigma, \beta, \gamma)=\frac{\alpha \frac{\beta}{\gamma}}{\frac{x}{\gamma}\left[\left(\frac{x}{\gamma}\right)^{\beta}+\alpha\right]}, \quad 0 \leq x \leq \infty .
$$

The next result provide the behavior of the RHR of the $\mathrm{M}-\mathrm{O} \log$ - $\operatorname{logistic}(\alpha, \beta, \gamma)$ distribution, and can be verified using elementary calculus.

Lemma 1 Let $X \sim M-O \log$-logistic $(\alpha, \beta, \gamma)$, then the reversed hazard rate is decreasing if $\beta>-1$, independent of $\alpha$ and $\gamma$.

Figure 1 illustrates some of the possible shapes of the reversed hazard rate functions of M-O $\log$-logistic $(\alpha, \beta, \gamma)$ distribution for different values of the parameter $\alpha$.

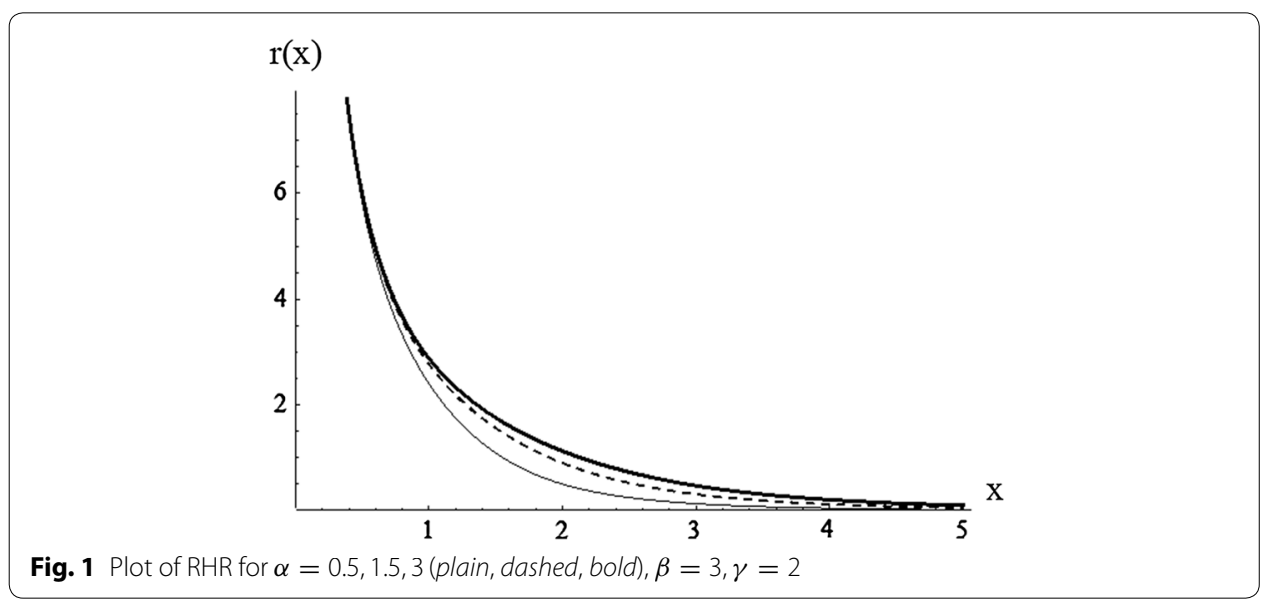


The conditional reliability function is a measure of the equipment's performance, indicating the probability of survival during a period of time, knowing that the equipment has not failed yet. This probability can be used to calculate the mean residual lifetime (MRL), and the optimal replacement policy for the equipment. If the probability is calculated while assuming that the equipment has not yet been put to work, it indicates the unconditional reliability of the equipment. When a condition monitoring system is available, analysts are interested in knowing the reliability based on the latest available information on the equipment's degradation state, i.e. the conditional reliability, while taking into consideration the information obtained from the condition monitoring system. The MRL function is very important in reliability and survival analysis because it describes the aging process. More specifically, if the random variable $X$ represents the life of a component, then MRL is given by

$$
\begin{aligned}
\mu(t) & =E[X-t \mid X>t] \\
& =\frac{1}{\bar{G}(t)} \int_{t}^{\infty} \bar{G}(x) d x, \quad t>0 .
\end{aligned}
$$

Although the MRL function is defined for any random variable $X$, it is of particular interest when $X$ is a non-negative random variable because it can then be thought of as a lifetime of a device, and then represents the conditional expected residual life of the device at time given that the device is still active at time $t$. In replacement and repair strategies, although the shape of the failure rate function plays an important role, the MRL function is found to be more relevant than the HR function because the former summarizes the entire residual life function, whereas the latter considers only the risk of instantaneous failure at some time. The MRL function of a random variable $X$ with $\mathrm{M}-\mathrm{O}$ $\log$-logistic is

$$
\mu(t)=\frac{t^{\beta}+\alpha \gamma^{\beta}}{\alpha \gamma^{\beta}} \int_{t}^{\infty} \frac{\alpha \gamma^{\beta}}{x^{\beta}+\alpha \gamma^{\beta}} d x .
$$

The value in Eq. (8) can be obtained numerically. Table 1 displays the mean residual life at point $t=2$ for M-O log-logistic at $\beta=3, \gamma=2$ and different choices of parameter $\alpha$.

From the above example, it is noted that the mean residual life is generally increasing for increasing values of $\alpha$ (Fig. 2).

Another interesting reliability function is the mean inactivity time (MIT) function (also known as the mean past lifetime and the mean waiting time functions). This function is well-known reliability measure which has several applications in many disciplines such as reliability theory, survival analysis, and actuarial studies. The MIT function of $X$ is defined by

Table 1 Mean residual life of $M-O$ log-logistic

\begin{tabular}{llll}
\hline $\boldsymbol{\alpha}$ & $\boldsymbol{\beta}$ & $\boldsymbol{\gamma}$ & MRL at $\boldsymbol{t}=\mathbf{2}$ \\
\hline 0.3 & 3 & 2 & 1.16808 \\
0.7 & 3 & 2 & 1.36339 \\
1.5 & 3 & 2 & 1.69029 \\
2.5 & 3 & 2 & 2.02493 \\
\hline
\end{tabular}




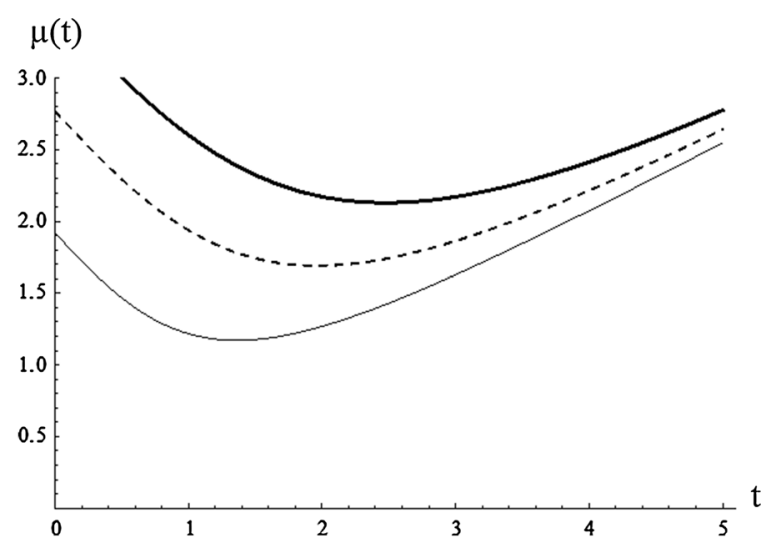

Fig. 2 Plot of MRL for $\alpha=0.5,1.5,3$ (plain, dashed, bold) , $\beta=3, \gamma=2$

$$
m(t)=\frac{1}{G(t)} \int_{0}^{t} G(x) d x, \quad t>0 .
$$

The MIT function of a random variable $X$ with $\mathrm{M}-\mathrm{O} \log$-logistic is

$$
m(t)=\frac{t^{\beta}+\alpha \gamma^{\beta}}{t^{\beta}} \int_{0}^{t} \frac{x^{\beta}}{x^{\beta}+\alpha \gamma^{\beta}} d x .
$$

The value of the function in (9) can be obtained by a numerical calculation. Table 2 displays the mean inactivity time at point $t=2$ for $\mathrm{M}-\mathrm{O} \log$ - $\operatorname{logistic}$ at $\beta=3, \gamma=2$ and different choices of parameter $\alpha$ (Additional file 2).

From the above example, it is noted that the mean inactivity time is generally decreasing for increasing values of $\alpha$ (Fig. 3).

Recently, a reliability measure called strong mean inactivity time (SMIT) function has been introduced and studied (see Kayid and Izadkhah 2014). The SMIT function of a random variable $X$ with $\mathrm{M}-\mathrm{O} \log$-logistic is

$$
\begin{aligned}
M_{T}(t) & =\frac{1}{G(t)} \int_{0}^{t} 2 x G(x) d x \\
& =\frac{2\left[t^{\beta}+\alpha \gamma^{\beta}\right]}{t^{\beta}} \int_{0}^{t} \frac{x^{\beta+1}}{x^{\beta}+\alpha \gamma^{\beta}} d x .
\end{aligned}
$$

Table 3 displays the strong mean inactivity time at point $t=2$ for $\mathrm{M}-\mathrm{O} \log$-logistic at $\beta=3, \gamma=2$ and different choices of parameter $\alpha$ (Additional file 3).

Table 2 Mean inactivity time of M-O log-logistic

\begin{tabular}{llll}
\hline $\boldsymbol{\alpha}$ & $\boldsymbol{\beta}$ & $\boldsymbol{\gamma}$ & MIT at $\boldsymbol{t}=\mathbf{2}$ \\
\hline 0.3 & 3 & 2 & 0.84578 \\
0.7 & 3 & 2 & 0.703955 \\
1.5 & 3 & 2 & 0.614498 \\
2.5 & 3 & 2 & 0.574372 \\
\hline
\end{tabular}




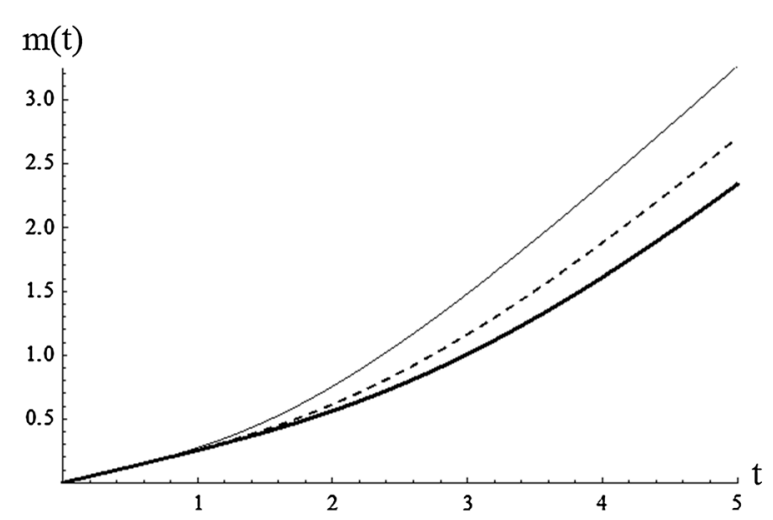

Fig. 3 Plot of MIT for $\alpha=0.5,1.5,3$ (plain, dashed, bold), $\beta=3, \gamma=2$ )

Table 3 Strong mean inactivity time of M-O log-logistic

\begin{tabular}{llll}
\hline $\boldsymbol{\alpha}$ & $\boldsymbol{\beta}$ & $\boldsymbol{\gamma}$ & SMIT at $\boldsymbol{t}=\mathbf{2}$ \\
\hline 0.3 & 3 & 2 & 2.48358 \\
0.7 & 3 & 2 & 2.14164 \\
1.5 & 3 & 2 & 1.911302 \\
2.5 & 3 & 2 & 1.804316 \\
\hline
\end{tabular}

From the above example, it is noted that the strong mean inactivity time is generally decreasing for increasing values of $\alpha$ (Fig. 4).

Mean, variance

We consider a random variable $X$ with $\mathrm{M}-\mathrm{O} \log$-logistic $(\alpha, \beta, \gamma)$. The mean and variance are given, respectively, by

$$
E(X)=\int_{0}^{\infty} \frac{x \alpha \frac{\beta}{\gamma}\left(\frac{x}{\gamma}\right)^{\beta-1}}{\left(\left(\frac{x}{\gamma}\right)^{\beta}+\alpha\right)^{2}} d x,
$$

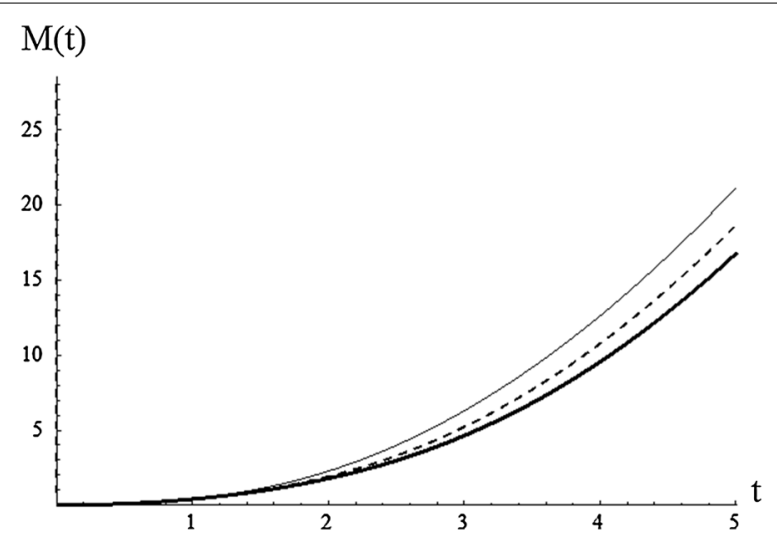

Fig. 4 Plot of SMIT for $\alpha=0.5,1.5,3$ (plain, dashed, bold), $\beta=3, \gamma=2$ 
and

$$
E\left(X^{2}\right)=\int_{0}^{\infty} \frac{x^{2} \alpha \frac{\beta}{\gamma}\left(\frac{x}{\gamma}\right)^{\beta-1}}{\left(\left(\frac{x}{\gamma}\right)^{\beta}+\alpha\right)^{2}} d x
$$

Hence

$$
\operatorname{Var}(X)=\int_{0}^{\infty} \frac{x^{2} \alpha \frac{\beta}{\gamma}\left(\frac{x}{\gamma}\right)^{\beta-1}}{\left(\left(\frac{x}{\gamma}\right)^{\beta}+\alpha\right)^{2}} d x-\left[\int_{0}^{\infty} \frac{x \alpha \frac{\beta}{\gamma}\left(\frac{x}{\gamma}\right)^{\beta-1}}{\left(\left(\frac{x}{\gamma}\right)^{\beta}+\alpha\right)^{2}} d x\right]^{2}
$$

In general, the last integrals cannot be given explicitly in terms of $\alpha, \beta, \gamma$. The mean $\mathrm{E}(\mathrm{X})$ and the variance $\operatorname{Var}(\mathrm{X})$ of $\mathrm{M}-\mathrm{O}$ log-logistic are shown graphically in Figs. 5 and 6 for different value of $\alpha$ and $\beta=3, \gamma=2$. These figures show the mean and variance increase as the value of $\alpha$ increases.

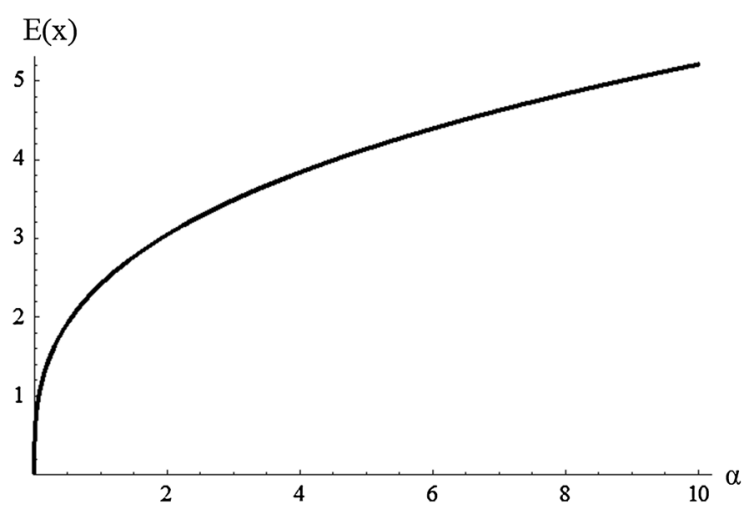

Fig. 5 Plot of the mean of M-O log-logistic for different value of $\alpha$ and $\beta=3, \gamma=2$

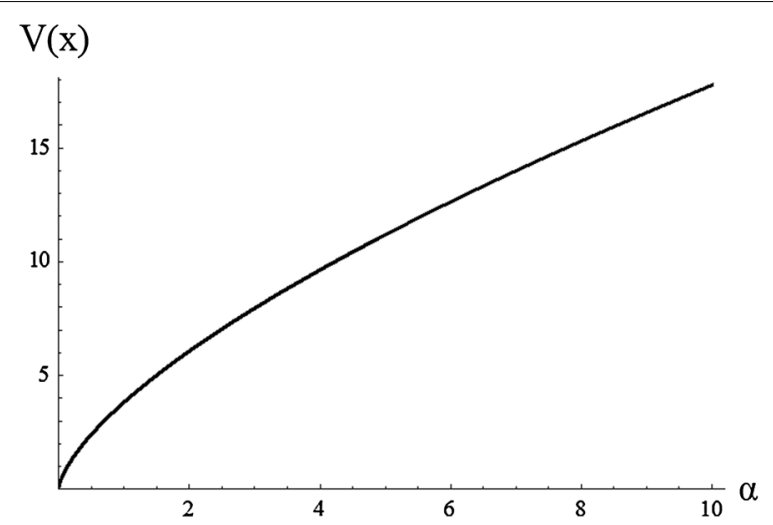

Fig. 6 Plot of the variance of $\mathrm{M}-\mathrm{O}$ log-logistic for different value of $\alpha$ and $\beta=3, \gamma=2$ 
Median

Let $X$ be a random variable with $\mathrm{M}-\mathrm{O} \log -\operatorname{logistic}(\alpha, \beta, \gamma)$. The median of this distribution is the value $m$ that satisfies

$$
\int_{0}^{m} \frac{\alpha \frac{\beta}{\gamma}\left(\frac{x}{\gamma}\right)^{\beta-1} d x}{\left[\left(\frac{x}{\gamma}\right)^{\beta}+\alpha\right]^{2}}=0.5
$$

Table 4 displays the median for $\mathrm{M}-\mathrm{O} \log$-logistic at $\beta=2, \gamma=0.2$ and different choices of parameter $\alpha$. It is noted that the median is generally increasing for increasing values of $\alpha$.

\section{Renyi entropy}

Entropy has been used in various situations in science and engineering. The entropy of a random variable $X$ with density function $g(x)$ is a measure of variation of the uncertainty. The Renyi entropy of order $\delta$ is defined by

$$
H_{\delta}(x)=\frac{1}{1-\delta} \log \left(\int_{-\infty}^{\infty} g(x)^{\delta} d x\right), \quad \delta \geq 0, \quad \delta \neq 1 .
$$

Let $X$ be a random variable with $\mathrm{M}-\mathrm{O} \log$-logistic $(\alpha, \beta, \gamma)$. The corresponding Renyi entropy is obtained as

$$
H_{\delta}(x)=\frac{1}{1-\delta} \log \left\{\int_{0}^{\infty}\left(\frac{\alpha \frac{\beta}{\gamma}\left(\frac{x}{\gamma}\right)^{\beta-1}}{\left[\left(\frac{x}{\gamma}\right)^{\beta}+\alpha\right]^{2}}\right)^{\delta} d x\right\}, \quad \delta \geq 0, \quad \delta \neq 1 .
$$

Table 5 displays the Renyi entropy for $\mathrm{M}-\mathrm{O} \log$-logistic at $\delta=3, \beta=1.5, \gamma=1$ and different choices of the parameter $\alpha$. It is noted that the Renyi entropy is generally increasing for increasing values of $\alpha$.

\section{Maximum likelihood estimators}

In statistics, maximum-likelihood estimation (MLE) is a method of estimating the parameters of a statistical model. When applied to a data set and given a statistical model, MLE provides estimates for the model's parameters. The method of maximum likelihood corresponds to many well-known estimation methods in statistics.

Let $X_{1}, \ldots, X_{n}$ be a random sample from $\mathrm{M}-\mathrm{O} \log$-logistic $(\alpha, \beta, \gamma)$, the likelihood function is given by

\section{Table 4 Median of M-O log-logistic}

\begin{tabular}{llll}
\hline $\boldsymbol{\alpha}$ & $\boldsymbol{\beta}$ & $\boldsymbol{\gamma}$ & Median \\
\hline 0.3 & 2 & 0.2 & 0.109545 \\
0.7 & 2 & 0.2 & 0.167332 \\
1.3 & 2 & 0.2 & 0.228035 \\
2 & 2 & 0.2 & 0.282843 \\
\hline
\end{tabular}


Table 5 Renyi entropy of M-O log-logistic

\begin{tabular}{llll}
\hline $\boldsymbol{\alpha}$ & $\boldsymbol{\beta}$ & $\boldsymbol{\gamma}$ & Renyi entropy \\
\hline 0.3 & 1.5 & 1 & 0.12391 \\
0.7 & 1.5 & 1 & 0.68877 \\
1.5 & 1.5 & 1 & 1.19687 \\
2 & 1.5 & 1 & 1.38866 \\
\hline
\end{tabular}

$$
L\left(X_{1}, \ldots, X_{n} \mid \alpha, \sigma, \beta, \gamma\right)=\frac{\alpha^{n}\left(\frac{\beta}{\gamma}\right)^{n}\left[\prod_{i=1}^{n}\left(\frac{x_{i}}{\gamma}\right)^{\beta-1}\right]}{\prod_{i=1}^{n}\left[\left(\frac{x_{i}}{\gamma}\right)^{\beta}+\alpha\right]^{2}} .
$$

The logarithm of the likelihood function is then given by

$$
\begin{aligned}
\ell\left(X_{1}, \ldots, X_{n} \mid \alpha, \sigma, \beta, \gamma\right)= & n \ln \alpha+n \ln \left(\frac{\beta}{\gamma}\right)+(\beta-1) \sum_{i=1}^{n} \ln \left(\frac{x_{i}}{\gamma}\right) \\
& -2 \sum_{i=1}^{n} \ln \left(\left(\frac{x_{i}}{\gamma}\right)^{\beta}+\alpha\right) .
\end{aligned}
$$

The maximum likelihood estimators (MLEs) of $\alpha, \beta$ and $\gamma$ can be obtained by solving the nonlinear equations

$$
\begin{aligned}
& \frac{\partial \ell}{\partial \alpha}=\frac{n}{\alpha}-2 \sum_{i=1}^{n} \frac{1}{\left(\frac{x_{i}}{\gamma}\right)^{\beta}+\alpha}=0 \\
& \frac{\partial \ell}{\partial \beta}=\frac{n}{\beta}+\sum_{i=1}^{n} \ln \left(\frac{x_{i}}{\gamma}\right)-2 \sum_{i=1}^{n} \frac{\left(\frac{x_{i}}{\gamma}\right)^{\beta} \ln \left(\frac{x_{i}}{\gamma}\right)}{\left(\frac{x_{i}}{\gamma}\right)^{\beta}+\alpha}=0 \\
& \frac{\partial \ell}{\partial \gamma}=-\frac{n}{\gamma}-\frac{n(\beta-1)}{\gamma}+2 \sum_{i=1}^{n} \frac{\beta x_{i}^{\beta}}{\left(\left(\frac{x_{i}}{\gamma}\right)^{\beta}+\alpha\right) \gamma^{\beta+1}}=0
\end{aligned}
$$

There is no explicit solution for Eqs. (16)-(18), so they need to be solved numerically. For a given known scale parameter $(\gamma=1), 1000$ different samples are simulated from $\mathrm{M}-\mathrm{O} \log$-logistic with different sizes and different values of the scale parameter $\alpha$. We studied the behavior of the MLEs from unknown scale parameter $\alpha$ and shape parameter $\beta$. The values of $\alpha$ are taken as $0.8,1.5$, and 2.5, while the value of $\beta$ is 2 . Tables 6 and 7 represent MLEs of parameter $\alpha$ and $\beta$, respectively.

From Table 6 it is observed that the estimate, mean square error and the bias of the MLE of the parameter $\alpha$ are decreasing when the sample size $(n)$ is increasing. From Table 7 it is observed that the estimate, mean square errors, and the bias of the MLE 
Table 6 MLE of the parameter $\alpha$

\begin{tabular}{lrlll}
\hline $\boldsymbol{\alpha}$ & $\boldsymbol{n}$ & Estimate & Bias & MSE \\
\hline 0.3 & 20 & 0.296275 & -0.00372454 & 0.0237631 \\
& 50 & 0.231197 & -0.0688027 & 0.0182828 \\
& 70 & 0.252118 & -0.047882 & 0.0128916 \\
& 150 & 0.285643 & -0.0143573 & 0.00492491 \\
1.2 & 20 & 1.02572 & -0.174276 & 0.291608 \\
& 50 & 1.20359 & 0.00358953 & 0.103632 \\
& 70 & 1.12847 & -0.0715284 & 0.119474 \\
& 150 & 1.19823 & -0.00177408 & 0.0339848 \\
2.5 & 20 & 2.34865 & -0.151351 & 1.2994 \\
& 50 & 2.59336 & 0.0933644 & 0.517638 \\
& 70 & 2.57025 & 0.0702524 & 0.392246 \\
& 150 & 2.53202 & 0.0320212 & 0.151326 \\
\hline
\end{tabular}

of the parameter $\beta$ are decreasing when the sample size $(n)$ is increasing. The second derivatives of (16)-(18) are

$$
\begin{aligned}
\frac{\partial^{2} \ell}{\partial \alpha^{2}} & =\frac{-n}{\alpha^{2}}+2 \sum_{i=1}^{n} \frac{1}{\left(\left(\frac{x_{i}}{\gamma}\right)^{\beta}+\alpha\right)^{2}} \cdot \\
\frac{\partial^{2} \ell}{\partial \beta^{2}} & =\frac{-n}{\beta^{2}}-2 \sum_{i=1}^{n} \frac{\left(\frac{x_{i}}{\gamma}\right)^{\beta}\left(\ln \left(\frac{x_{i}}{\gamma}\right)\right)^{2}\left(\left(\frac{x_{i}}{\gamma}\right)^{\beta}+\alpha\right)-\left(\left(\frac{x_{i}}{\gamma}\right)^{\beta} \ln \left(\frac{x_{i}}{\gamma}\right)\right)^{2}}{\left(\left(\frac{x_{i}}{\gamma}\right)^{\beta}+\alpha\right)^{2}} . \\
\frac{\partial^{2} \ell}{\partial \gamma^{2}} & =\frac{n}{\gamma^{2}}+\frac{n(\beta-1)}{\gamma^{2}}+2 \sum_{i=1}^{n} \frac{\beta x_{i}^{\beta}\left[\beta x_{i}^{\beta}-(\beta+1) \gamma^{\beta}\left(\left(\frac{x_{i}}{\gamma}\right)^{\beta}+\alpha\right)\right]}{\left(\left(\frac{x_{i}}{\gamma}\right)^{\beta}+\alpha\right)^{2} \gamma^{2(\beta+1)}} \\
\frac{\partial^{2} \ell}{\partial \alpha \partial \beta} & =2 \sum_{i=1}^{n} \frac{\left(\frac{x_{i}}{\gamma}\right)^{\beta} \ln \left(\frac{x_{i}}{\gamma}\right)^{\beta}}{\left(\left(\frac{x_{i}}{\gamma}\right)^{\beta}+\alpha\right)^{2}} . \\
\frac{\partial^{2} \ell}{\partial \alpha \partial \gamma} & =-2 \sum_{i=1}^{n} \frac{\beta x_{i}^{\beta}}{\left(\left(\frac{x_{i}}{\gamma}\right)^{\beta}+\alpha\right)^{2} \gamma^{\beta+1}} . \\
\frac{\partial^{2} \ell}{\partial \beta \partial \gamma} & =-\frac{n}{\gamma}+2 \sum_{i=1}^{n} \frac{\left(\left(\frac{x_{i}}{\gamma}\right)^{\beta}+\alpha\right)\left(\frac{\beta x_{i}^{\beta} \ln \left(\frac{x_{i}}{\gamma}\right)+x_{i}^{\beta}}{\gamma^{\beta+1}}\right)^{\beta x_{i}^{2 \beta} \ln \left(\frac{x_{i}}{\gamma}\right)}}{\gamma^{2 \beta+1}}
\end{aligned}
$$

If we denote the MLE of $\theta=(\alpha, \beta, \gamma)$ by $\hat{\theta}=(\hat{\alpha}, \hat{\beta}, \hat{\gamma})$, the observed information matrix is then given by 
Table 7 MLE of the parameter $\beta$

\begin{tabular}{lrlcl}
\hline $\boldsymbol{\beta}$ & $\boldsymbol{n}$ & Estimate & Bias & MSE \\
\hline$\alpha=0.3$ & 20 & 1.91064 & -0.0893629 & 0.394056 \\
& 50 & 2.02933 & 0.0293271 & 0.0744106 \\
& 70 & 1.9975 & -0.00249889 & 0.0850081 \\
$\alpha=1.2$ & 150 & 2.01348 & 0.0134826 & 0.0190133 \\
& 20 & 1.96364 & -0.036358 & 0.341544 \\
& 50 & 2.03782 & 0.0378182 & 0.0577503 \\
& 70 & 2.02835 & 0.0283514 & 0.0409679 \\
$\alpha=2.5$ & 150 & 2.01348 & 0.0134826 & 0.0190133 \\
& 20 & 1.94389 & -0.0561107 & 0.372105 \\
& 50 & 1.9975 & -0.00249889 & 0.0850081 \\
& 70 & 2.02563 & 0.0283514 & 0.0409679 \\
& 150 & 2.01095 & 0.0109501 & 0.0227298 \\
\hline
\end{tabular}

$$
I(\theta)=\left[\begin{array}{rrr}
-\frac{\partial^{2} \ell}{\partial \alpha^{2}} & -\frac{\partial^{2} \ell}{\partial \alpha \partial \beta} & -\frac{\partial^{2} \ell}{\partial \alpha \partial \gamma} \\
-\frac{\partial^{2} \ell}{\partial \alpha \partial \beta} & -\frac{\partial^{2} \ell}{\partial \beta^{2}} & -\frac{\partial^{2} \ell}{\partial \beta \partial \gamma} \\
-\frac{\partial^{2} \ell}{\partial \alpha \partial \gamma} & -\frac{\partial^{2} \ell}{\partial \beta \partial \gamma} & -\frac{\partial^{2} \ell}{\partial \gamma^{2}}
\end{array}\right]
$$

Hence the variance covariance matrix would be $I^{-1}(\theta)$. The approximate $(1-\delta) 100 \%$ confidence intervals (CIs) for the parameters $\alpha, \beta$ and $\gamma$ are $\hat{\alpha} \pm Z_{\frac{\delta}{2}} V(\hat{\alpha}), \hat{\beta} \pm Z_{\frac{\delta}{2}} V(\hat{\beta})$ and $\hat{\gamma} \pm Z_{\frac{\delta}{2}} V(\hat{\gamma})$ respectively, where $V(\hat{\alpha}), V(\hat{\beta})$ and $V(\hat{\gamma})$ are the variances of $\hat{\alpha}, \hat{\beta}$ and $\hat{\gamma}$, which are given by the diagonal elements of $I^{-1}(\theta)$, and $Z_{\frac{\delta}{2}}$ is the upper $(\delta / 2)$ percentile of standard normal distribution.

\section{Fitting reliability data}

In this section, we provide two data sets analysis to show how the model works in practice.

\section{First data set}

The first data set given in Gupta et al. (1999) is about days of survival for lung cancer patients

Data set

\begin{tabular}{rrrrrrrrrr}
\hline 389 & 18 & 22 & 10 & 112 & 63 & 100 & 13 & 151 & 467 \\
162 & 117 & 122 & 33 & 42 & 99 & 283 & 80 & 314 & 112 \\
\hline
\end{tabular}

Some properties of the data set were computed in Table 8.

From the above table, it is clear that the distribution of this data set is positively skewed right and leptokurtic. The parameter of the sample is estimated numerically. We used Eqs. (16)-(18) to obtain MLEs estimate and the results are given in Table 9.

Table 8 Some properties of data set

\begin{tabular}{llll}
\hline$E(X)$ & $\operatorname{Var}(X)$ & Kurtosis & Skewness \\
\hline 135.45 & $16,735.1$ & 0.526142 & 1.26934 \\
\hline
\end{tabular}


Table 9 MLE for data set

\begin{tabular}{lc}
\hline Parameter & MLE \\
\hline$\alpha$ & 10.833 \\
$\beta$ & 1.58621 \\
$\gamma$ & 19.7451 \\
\hline
\end{tabular}

If we want to test if this data fits the $\mathrm{M}-\mathrm{O} \log$-logistic $(\alpha, \beta, \gamma)$, our hypotheses is $H_{0}: F=F_{\mathrm{M}-\mathrm{O} \log -\log i s t i c}$ versus $H_{1}: F \neq F_{\mathrm{M}-\mathrm{O} \log \text {-logistic }}$. We use the Kolmogorov-Smirnov $(K-S)$ distances between the empirical distribution function and the fitted distribution function to determine the appropriateness of the model. $K-S$ at $95 \%$ CIs value and the corresponding $p$ value are presented in Table 10 .

The small $\mathrm{K}-\mathrm{S}$ distance and the large $p$ value for the test indicate this data fits the $\mathrm{M}-\mathrm{O}$ log-logistic quite well. Also we use likelihood ratio test (LRT) to determine the appropriateness of the model. The hypotheses are as follows:

$$
H_{0}: \alpha=1(\log \text {-logistic }) \text { versus } H_{1}: \alpha \neq 1(M-O \log \text {-logistic }) .
$$

The log-likelihood value, likelihood ratio statistic $(\Lambda)$ and corresponding $p$ value are presented in Table 11.

We note that the calculated LRT statistic is greater than the critical point for this test, which is 6.635 , and also that the $p$ value is very small. According to the LRT, we conclude that this data fits the $\mathrm{M}-\mathrm{O} \log$-logistic much better than the log-logistic distribution.

\section{Second data set}

The second data set obtained from www.isixigma.com represents a cycle time of a process.

Data set

\begin{tabular}{|c|c|c|c|c|c|c|c|c|c|c|c|c|c|}
\hline 10 & 13 & 13 & 14 & 14 & 15 & 15 & 16 & 25 & 26 & 26 & 27 & 38 & 53 \\
\hline 17 & 17 & 17 & 17 & 18 & 18 & 18 & 19 & 27 & 27 & 28 & 28 & 42 & \\
\hline 21 & 21 & 21 & 22 & 22 & 23 & 24 & 25 & 30 & 34 & 35 & 35 & 42 & \\
\hline
\end{tabular}

Some properties of the data set were computed in Table 12.

From the above table, it's clear that the distribution of this data set is positively skewed right and leptokurtic. The parameter of the sample is estimated numerically. We used Eqs. (16)-(18) to obtain MLEs estimate and the results are given in Table 13.

We want to test if these data fit the $\mathrm{M}-\mathrm{O} \log$-logistic or not, our hypotheses is $H_{0}: F=F_{\mathrm{M}-\mathrm{O} \log \text {-logistic }}$ versus $H_{1}: F \neq F_{\mathrm{M}-\mathrm{O} \log \text {-logistic }}$. We use the $K-S$ distances

Table 10 The $K-S$ and $p$ value of data set

\begin{tabular}{lc}
\hline $\boldsymbol{K}-\boldsymbol{S}$ & $\boldsymbol{p}$ value \\
\hline 0.103124 & 0.131 \\
\hline
\end{tabular}

Table 11 The result of likelihood ratio test

\begin{tabular}{lll}
\hline Log-likelihood & $\boldsymbol{\Lambda}$ & $\boldsymbol{p}$ value \\
\hline-118.851 & 32.7814 & $1.03127 \times 10^{-8}$ \\
\hline
\end{tabular}


Table 12 Some properties of data set

\begin{tabular}{llll}
\hline$E(X)$ & $\operatorname{Var}(X)$ & Kurtosis & Skewness \\
\hline 23.825 & 86.1481 & 0.934659 & 1.05974 \\
\hline
\end{tabular}

Table 13 MLE for data set

\begin{tabular}{lc}
\hline Parameter & MLE \\
\hline$\alpha$ & 0.457038 \\
$\beta$ & 4.70536 \\
$\gamma$ & 26.0741 \\
\hline
\end{tabular}

between the empirical distribution function and the fitted distribution function to determine the appropriateness of the model. $K-S$ value at $99 \%$ CIs and the corresponding $p$ value are presented in Table 14.

The small K-S distance and the large p-value for the test indicate that this data fits the $\mathrm{M}-\mathrm{O} \log$-logistic quite well. Also we use LRT to determine the appropriateness of the model. The hypotheses are as follow:

$$
H_{0}: \alpha=1(\log \text {-logistic }) \quad \text { versus } \quad H_{1}: \alpha \neq 1(\text { MOlog-logistic }) .
$$

Log-likelihood value, likelihood ratio statistic $(\Lambda)$ and corresponding $p$ value are presented in Table 15.

We note that the calculated LRT statistic is greater than the critical point for this test, which is 6.635 , and also that the $p$ value is very small. According to the LRT, we conclude this data fits the $\mathrm{M}-\mathrm{O} \log$-logistic much better than the log-logistic distribution.

\section{Conclusion}

In this paper, an extended model based on log-logistic distribution is investigated. Some reliability and statistical properties of this model are obtained. Through numerical simulation, the MLE of the parameters are calculated and discussed. Finally, two sets of real data are fitted to this model and is shown to be appropriate. Further properties and applications of the model can be considered in the future of this research. In particular, the following topics are interesting and still remain as open problems:

1. Discuss the Bayesian analysis of the model.

2. Introduce and study a new class of weighted $\mathrm{M}-\mathrm{O}$ bivariate log-logistic distribution.

Table 14 The $K-S$ and $p$ value of data set

\begin{tabular}{lc}
\hline $\boldsymbol{K}-\boldsymbol{S}$ & $\boldsymbol{p}$ value \\
\hline 0.0982563 & 0.212 \\
\hline
\end{tabular}

Table 15 The result of likelihood ratio test

\begin{tabular}{lll}
\hline Log-likelihood & $\boldsymbol{\Lambda}$ & $\boldsymbol{p}$ value \\
\hline-141.405 & 7.84139 & 0.00510632 \\
\hline
\end{tabular}




\section{Additional files}

Additional file 1. First data set.

Additional file 2. Second data set.

Additional file 3. Simulation results.

Authors' contributions

All authors contributed equally to this work. All authors read and approved the final manuscript.

\section{Acknowledgements}

This research project was supported by a grant from the "Research Center of the Female Scientific and Medical Colleges", Deanship of Scientific Research, King Saud University.

\section{Competing interests}

All authors declare that there is no competing interests regarding the publication of this paper.

Received: 22 November 2015 Accepted: 15 March 2016

Published online: 31 March 2016

\section{References}

Alshangiti AM, Kayid M, M Almulhim (2016) Reliability analysis of extended generalized inverted exponential distribution with applications. J Syst Eng Electron (in press)

Alshangiti AM, Kayid M, Alarfaj B (2014) A new family of Marshall-Olkin extended distributions. J Comput Appl Math 271:369-379

Burr IW (1942) Cumulative frequency functions. Ann Math Stat 13:215-232

Cordeiro GM, Lemonte AJ (2012) On the Marshall-Olkin extended Weibull distribution. Stat Pap. doi:10.1007/ s00362-012-0431-8

El-Bassiouny AH, Abdo NF (2009) Reliability properties of extended Makeham distribution. Comput Methods Sci Technol 15:143-149

El-Bassiouny AH, Abdo NF (2010) Reliability properties of seven parameters Burr XII distribution. Comput Methods Sci Technol 16:127-133

Ghitany ME, Al-Awadhi FA, Alkhalfan LA (2007) Marshall-Olkin extended Lomax distribution and its application to censored data. Commun Stat Theory Methods 36:1855-1866

Gui W (2013) Marshall Olkin extended log logistic distribution and its application in minification process. Appl Math Sci 7:3947-3961

Gupta RC, Akman O, Lvin S (1999) A study of loglogistic model in survival analysis. Biom J 41:431-443

Jose K, Krishna E (2011) Marshall-Olkin extended uniform distribution. Probab Stat Forum 4:78-88

Kayid M, Izadkhah S (2014) Mean inactivity time function, associated orderings and classes of life distribution. IEEE Trans Reliab 63:593-602

Lin J, Li X (2012) Multivariate generalized Marshall-Olkin distributions and copulas. Methodol Comput Appl Probab. doi:10.1007/s11009-012-9297-4

Marshall AW, Olkin I (1997) A new method for adding a parameter to a family of distributions with application to the exponential and Weibull families. Biometrika 84:641-652

Mielke PW, Johnson ES (1973) Three-parameter kappa distribution maximum likelihood estimates and likelihood ratio tests. Mon Weather Rev 101:701-709

Okasha H, Kayid M (2016) A new family of Marshall-Olkin extended generalized linear exponential distribution. J Comput Appl Math 296:576-592

Ristic M, Jose K, Ancy J (2007) A Marshall-Olkin gamma distribution and minification process. Stress Anxiety Res Soc 11:107-117

Srinivasa GR, Ghitany ME, Kantam RL (2011) An economic reliability test plan for Marshall-Olkin extended exponential distribution. Appl Math Sci 5:103-112 